\title{
A concordance scenario for the observed neutrino from a tidal disruption event
}

\author{
Walter Winter ${ }^{1 \times}$ and Cecilia Lunardini $\mathbb{B}^{2}$
}

\begin{abstract}
During a tidal disruption event, a star is torn apart by the tidal forces of a supermassive black hole, with about $50 \%$ of the star's mass eventually accreted by the black hole. The resulting flare can, in extreme cases of super-Eddington mass accretion, result in a relativistic jet ${ }^{1-4}$. While tidal disruption events have been theoretically proposed as sources of high-energy cosmic rays $^{5,6}$ and neutrinos ${ }^{7-14}$, stacking searches indicate that their contribution to the diffuse extragalactic neutrino flux is very low ${ }^{15}$. However, a recent association of a track-like astrophysical neutrino (IceCube-191001A ${ }^{16}$ ) with a tidal disruption event (AT2019 $\mathrm{dsg}^{17}$ ) indicates that some tidal disruption events can accelerate cosmic rays to petaelectronvolt energies. Here we introduce a phenomenological concordance scenario with a relativistic jet to explain this association: an expanding cocoon progressively obscures the X-rays emitted by the accretion disk, while at the same time providing a sufficiently intense external target of backscattered X-rays for the production of neutrinos via proton-photon interactions. We also reproduce the delay (relative to the peak) of the neutrino emission by scaling the production radius with the black-body radius. Our energetics and assumptions for the jet and the cocoon are compatible with expectations from numerical simulations of tidal disruption events.
\end{abstract}

On 1 October 2019, a track-like astrophysical neutrino (named IceCube-191001A) was detected ${ }^{16}$; a dedicated multimessenger follow-up programme revealed the tidal disruption event (TDE) AT2019dsg as a candidate source, with a $P$ value of $0.2 \%$ to $0.5 \%$ of random association ${ }^{17}$, corresponding to $\sim 3 \sigma$ significance. The neutrino followed the peak of the AT2019dsg light curve by $t-t_{\text {peak }}=154 \mathrm{~d}$ and had a most likely energy $E \approx 0.2 \mathrm{PeV}$ (ref. ${ }^{16}$ and links therein). Its observation reveals a new class of cosmic ray sources, as it indicates that some TDEs can accelerate cosmic rays to petaelectronvolt energies.

The TDE AT2019dsg is located at redshift $z \approx 0.05$, or luminosity distance $d_{\mathrm{L}} \approx 230 \mathrm{Mpc}$. It was discovered in the optical-ultraviolet (UV) bands by the Zwicky Transient Facility (ZTF) on 9 April $2019^{18}$, and it reached its luminosity peak in this band on 30 April $2019\left(t_{\text {peak }}=58603\right.$ modified Julian date $)$. Several follow-up observations were conducted in the optical-UV ${ }^{18}$, radio $^{17,19,20}$ and X-ray ${ }^{17,21,22}$ bands, the latter starting at $t-t_{\text {peak }}=17 \mathrm{~d}$. The picture that emerged from the observations shows a several-months-long flare, with black body (BB) spectra observed in both the optical-UV (temperature $T_{\mathrm{BB}}=38,900 \mathrm{~K}$, photospheric radius $R_{\mathrm{BB}} \approx 5 \times 10^{14} \mathrm{~cm}$ ) and X-ray $\left(T_{\mathrm{x}} \approx 0.06 \mathrm{keV}, R_{\mathrm{x}} \approx 3 \times 10^{11}-7 \times 10^{11} \mathrm{~cm}\right)$ bands, and luminosities $L$ exponentially decaying over an (initial) timescale of $57.5 \mathrm{~d}$ and $10.3 \mathrm{~d}$ starting at $L_{\mathrm{BB}}=2.88 \times 10^{44} \mathrm{erg} \mathrm{s}^{-1}$ and $L_{\mathrm{X}} \approx 2.5 \times 10^{43} \mathrm{erg} \mathrm{s}^{-1}$, respectively (Fig. 1, thick black and blue curves). The quoted X-ray luminosity is for an energy window $[0.3-8.0] \mathrm{keV}$, whereas an X-ray luminosity $L_{\mathrm{X}} \approx 4 \times 10^{44} \mathrm{erg} \mathrm{s}^{-1}$ was found in ref. ${ }^{22}$ in the energy window [0.1-10] keV. Instead, the luminosity in radio emission was approximately constant over a nearly $90 \mathrm{~d}$ period, with increasing radius of emission $R_{\text {radio }}=\mathcal{O}\left(10^{16}\right) \mathrm{cm}$ (ref. ${ }^{17}$ ). The radio emission has been interpreted as an indication for a mildly relativistic outflow present over the timescale of the neutrino event. Furthermore, optical polarimetry observations of this TDE cannot be uniquely interpreted, and may provide some hint for a relativistic jet ${ }^{23}$. A further noteworthy element is that out of the 17 TDEs in the ZTF sample, only 4 were found to have a counterpart in X-rays; of these, AT2019dsg was the one with the highest sustained (over several days) X-ray luminosity.

In this study, we propose a coherent 'concordance' framework of a (dark or hidden) jetted TDE, which is consistent with the unified model (based on magnetohydrodynamical simulations) of Dai et al. ${ }^{24}$; see Methods for details. The framework describes the neutrino energy and arrival time-where the latter is somewhat a challenge-considering the overall decreasing trend of the multimessenger luminosities (Fig. 1, thick solid black and blue curves). A schematic concept is given in Fig. 2. At early times, the X-rays are visible for the observer, whose line of sight is on (or close to) the jet axis. Plasma shells collide at radial distance $R_{\mathrm{C}}$, where (internal) shocks form, leading to proton acceleration. We assume that absorption by the expanding outflow causes the exponential decay of the observed X-ray flux (Fig. 1, thick blue curve). Then, the same effect leads to photons isotropizing on the same timescale, which are backscattered and Doppler-boosted into the jet frame where they serve as targets for the neutrino production (Fig. 2, right panel). The physical jet power is taken from ref. ${ }^{24}$, and is assumed to follow the BB luminosity (Fig. 1, thick green curve). The jet ceases when the physical power drops below the Eddington luminosity. We assume that $R_{\mathrm{C}}$ evolves similarly to $R_{\mathrm{BB}}$, which is observed to decrease slightly over time. A decreasing $R_{\mathrm{C}}$ implies an increasingly compact collision region, and thus an increase of the neutrino production efficiency $\propto R_{\mathrm{C}}^{-2}$ at late times. Similarly, a larger $R_{\mathrm{C}}$, such as may be expected for a larger supermassive black hole $(\mathrm{SMBH})$ mass, would lead to a decreased neutrino production efficiency.

The result for the time evolution of the neutrino luminosity, $L_{\nu}$, is shown in Fig. 1 (red curve). Its initial rise and peak, at $t-t_{\text {peak }} \approx 30-70 \mathrm{~d}$, follows the isotropized target X-ray flux. At later times, the evolution of $L_{\nu}$ is mostly determined by the competition of the decreasing proton injection and target photon densities (driving a decrease of the neutrino flux) and the decreasing production radius $R_{\mathrm{C}}$ enhancing the neutrino flux. As a consequence of this interplay, the neutrino luminosity has a second peak at $t-t_{\text {peak }} \approx 130-170 \mathrm{~d}$. The late-time luminosity revival could contribute to explaining the observed detection of one neutrino

'Deutsches Elektronen-Synchrotron (DESY), Zeuthen, Germany. ${ }^{2}$ Department of Physics, Arizona State University, Tempe, AZ, USA.

凶e-mail:walter.winter@desy.de 


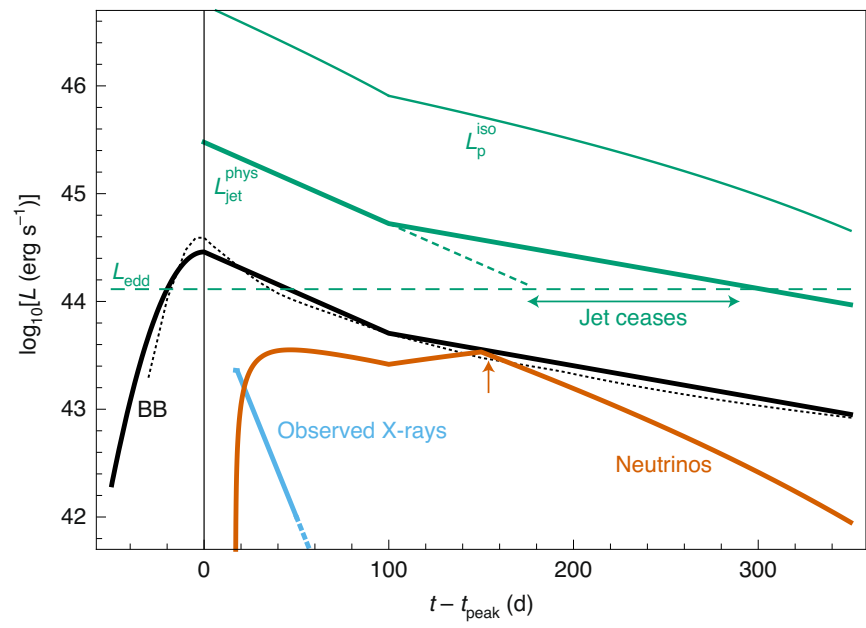

Fig. 1 | Time evolution of different luminosities in the jetted TDE model. The labels are directly given next to the curves; see main text for definitions. The neutrino luminosity is a result of our work, whereas the other curves are input quantities of the model. Thick black and blue (solid) curves (the latter starting at $t-t_{\text {peak }} \approx 17 \mathrm{~d}$, reflecting the lack of X-ray data before that point) are chosen to roughly follow data ${ }^{18}$; the dotted black curve represents a power-law fit from ref. ${ }^{18}$, which has a fixed late-time $t^{-5 / 3}$ behaviour. All shown luminosities are isotropic equivalent, and refer to the source/engine frame, except for $L_{\text {Edd }}$ and $L_{\text {jet }}^{\text {phys }}$. The vertical arrow marks the arrival time of the observed neutrino event.

at $t-t_{\text {peak }}=154 \mathrm{~d}$. Eventually, after the revival, $L_{\nu}$ undergoes a sharp drop from the jet cessation or the $R_{\mathrm{C}}$ stagnation (Methods and equation (5)).

Figure 3 shows the predicted neutrino fluence, $\mathcal{F}_{\mu}$, as well as two differential limits on the same quantity (see caption), for comparison. Compared with other cases of proton scattering on thermal
$\mathrm{X}$-rays, the neutrino energy spectrum is relatively wide here due to multipion processes dominating the neutrino production (see, for example, ref. ${ }^{25}$ for a similar case). The most likely value of the neutrino energy $\left(E_{\nu} \approx 0.2 \mathrm{PeV}\right.$, with a large uncertainty allowing up to one order of magnitude larger values), falls near the maximum of the fluence.

The total, time-integrated number of events predicted in IceCube, $N_{\iota}$, depends on the effective area used. We find $N_{\iota} \approx 0.26$ when using the point source effective area (which applies to a transient point source analysis), and $N_{\nu} \approx 0.05$ when using the gamma-ray follow-up effective area, which includes the probability that the alert system is triggered. Note that the observation of one event is well compatible with $N_{\nu} \ll 1$, due to Poissonian statistics and due to the Eddington bias ${ }^{26}$. From Fig. 3, we also observe that the early- and late-term contributions to the total fluence are comparable, which implies that a neutrino detection $\sim 150 \mathrm{~d}$ after the peak is plausible.

Let us discuss our proposed jet scenario in the broader context. Compared with the best-known jetted TDE, Swift J1644+57, AT2019dsg is very different: it is $\sim 10^{3}$ times less powerful (from the observer's point of view) in X-rays, and its X-ray spectrum is thermal, in contrast with the non-thermal spectrum of Swift J1644+57, which was interpreted as signature of a jet. Therefore, the existence of a jet in AT2019dsg might be less obvious, and leaves some open questions.

One of these is how to reconcile the non-observation of (non-thermal) jet signatures in X-rays and gamma rays-at least gamma rays in the $0.1-1.0 \mathrm{PeV}$ energy range, which are a direct counterpart of the neutrinos-with the expectation that the jet should be able to break out of a surrounding envelope material ${ }^{8}$ and therefore should not be completely hidden.

Currently, the sparseness of the data from AT2019dsg (for example, gamma-ray limits are relatively weak, see ref. ${ }^{17}$ ), does not allow for a clear description of the electromagnetic spectrum from the jet over a wide energy range. With regard to the microphysics in the jet, there is no evidence for non-thermal signatures from accelerated electrons, such as synchrotron radiation and inverse Compton scattering. This fact may indicate a relatively high baryonic loading
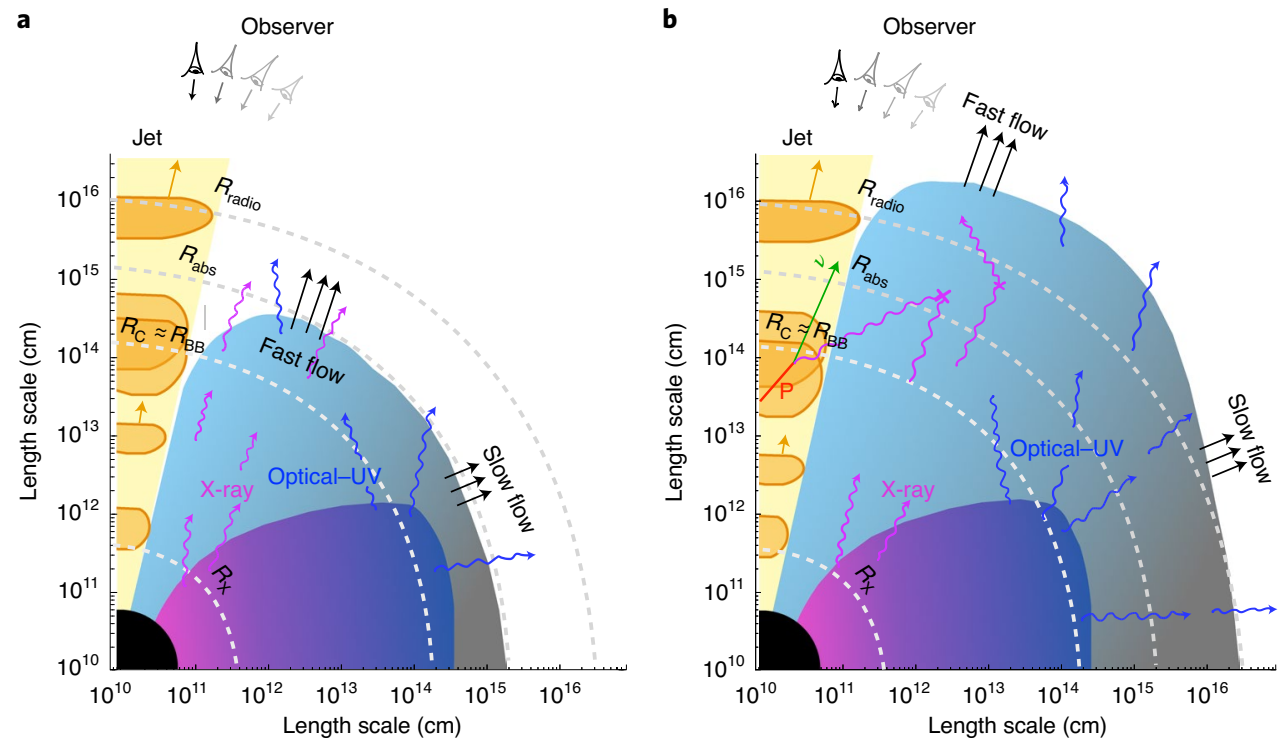

Fig. 2 | Illustration of the evolution of the TDE outflow in the concordance scenario. a,b, Here two time periods are shown: early $\left(t-t_{\text {peak }}<17 \mathrm{~d}\right)$, where $X$-rays can efficiently escape (a), and late ( $\left.t-t_{\text {peak }} \gg 17 \mathrm{~d}\right)$, where X-rays are absorbed/backscattered into the jet frame (b). The relevant length scales are marked as dashed lines to guide the eye (order magnitude only; as follows: $R_{\mathrm{X}}$, X-ray photosphere radius; $R_{\mathrm{BB}}$, $\mathrm{BB}$ radius; $R_{\text {radio, }}$ radio emission region; $R_{\text {abs' }}$ X-ray mean free path; $R_{C}$, neutrino production radius). The anticipated direction of the observer is shown as well. The proton acceleration and neutrino production is expected to happen at $R_{\mathrm{C}} \approx R_{\mathrm{BB}}$, where plasma shells collide and shocks form. The flow expands slightly faster in the direction of the jet axis. P, production region. 


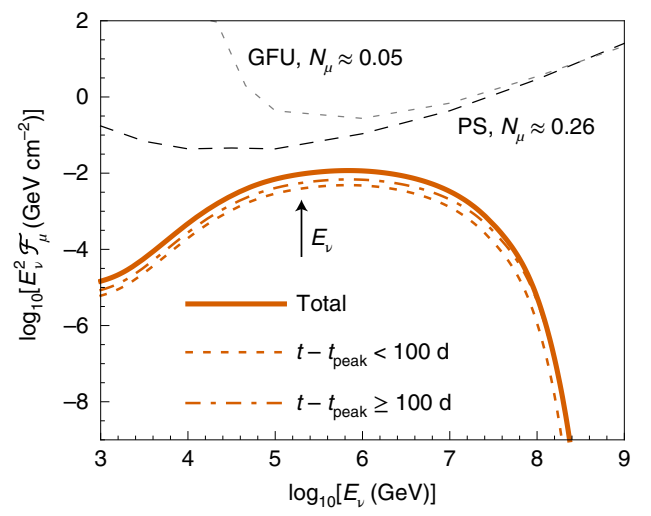

Fig. 3 | Predicted neutrino fluence for the jetted TDE model. The curves are computed as integrated muon neutrino and antineutrino fluence (including flavour mixing). The integrated contributions from early (red dashed) and late times (red dot-dashed) are shown separately. In comparison, the differential limits and predicted event rates using the gamma-ray follow-up (GFU ${ }^{52}$ ) and point source ( $\mathrm{PS}^{53}$ ) effective areas for the declination of AT2019dsg are shown; the likely neutrino energy is taken from ref. ${ }^{17}$. Here the differential limit (DL) is given by $E_{\nu}^{2} \mathcal{F}_{\mu}^{\mathrm{DL}}=E_{\nu} /\left(A_{\text {eff }}\left(E_{\nu}\right) \ln 10\right)$ as a function of the effective area $A_{\text {eff }}$ which implies that following the differential limit curve precisely for one order of magnitude in energy yields one neutrino event.

(energy in protons versus electromagnetic radiation) or unexpected parameters of the electron population in the jet, very different from Swift J1644+57. Our model does not require any assumptions on these quantities, as the input on the X-rays, which serve as target photons, is from observation.

Other explanations of the lack of jet signatures could be in the macroscopic picture, such as a possible intermittent nature of the jet, effects of an off-axis line of sight, a larger efficiency of energy dissipation in the collision region or an unusual jet geometry-as there may be effects from jet recollimation, twisting or precession. Propagation effects could explain the suppression of petaelectronvolt gamma rays, which may be reprocessed in the source or absorbed in the extragalactic background light. More information on a possible jet might be obtained by very long baseline radio interferometry or by late-term radio observations ${ }^{27}$ if the observed radio signal is interpreted as the afterglow of a relativistic jet; see also ref. ${ }^{28}$ for further discussions.

We have described the observation of a neutrino coincident with the tidal disruption event AT2019dsg in a jetted TDE model. In our interpretation, the unusually high X-ray luminosity of AT2019dsg is the reason for the efficient neutrino production, which implies that X-ray-bright TDEs might also be neutrino bright. We have also shown that the late time of the neutrino signal (about $150 \mathrm{ds}$ after the optical peak) is not a coincidence if the neutrino production radius scales with the $\mathrm{BB}$ radius, whereas a very early neutrino signal close to the peak is not expected because the X-rays have not isotropized yet. Energetics and parameters match a unified TDE model from numerical simulations ${ }^{24}$, which have led to our concordance model. Indeed, for a large enough black hole spin, a jet may be expected in a unified picture of TDEs in addition to a mildly relativistic outflow ${ }^{24}$, which has been (indirectly) observed ${ }^{17}$. A preliminary support to the jet hypothesis also comes from optical polarimetry of AT2019 $\mathrm{dsg}^{23}$.

If AT2019dsg and Swift J1644+57 are both jetted TDEs, then one will have to conclude that the phenomenology of TDEs is very diverse. New dedicated studies will be needed to explain this variety in terms of parameters such as the black hole mass and spin, the type of disrupted star, the type of star-black hole approach trajectory, and the spectral energy distribution. The diversity will then impact the estimate of the diffuse flux of neutrinos from TDEs. Our calculations show that AT2019dsg-like TDEs could contribute to the total neutrino flux observed at IceCube at the percent level.

We conclude that TDEs might be a promising class of neutrino emitters. While we have presented only one model here, other possibilities are conceivable, such as the interaction of an isotropic outflow with UV photons ${ }^{17}$, non-relativistic shocks forming in the environment ${ }^{29}$ or a neutrino production from the accretion disk itself, especially radiatively inefficient accretion flows or magnetically arrested disk states ${ }^{14}$; the neutrino production may also happen in a hot corona similar to that of an active galactic nucleus $(\mathrm{AGN})^{30}$. Our model is unique in that we have emphasized the connection to the X-ray observations, we have described the late, post-peak neutrino observation, and we have obtained a sufficiently high neutrino fluence to describe the observations in spite of a relatively small assumed SMBH mass.

\section{Methods}

A star (of mass $m$ ) is disrupted by a SMBH (mass $M$ ) if (1) it falls within a distance less than the tidal radius:

$$
r_{\mathrm{t}} \approx 9 \times 10^{12} \mathrm{~cm}\left(\frac{M}{10^{6} M_{\odot}}\right)^{1 / 3} \frac{R}{R_{\odot}}\left(\frac{m}{M_{\odot}}\right)^{-1 / 3}
$$

and (2) the tidal radius exceeds the SMBH Schwarzschild radius

$$
R_{\mathrm{s}}=\frac{2 M G}{c^{2}} \approx 3 \times 10^{11} \mathrm{~cm}\left(\frac{M}{10^{6} M_{\odot}}\right)
$$

(where $G$ is the gravitational constant and $c$ is the speed of light) as otherwise the star is swallowed by the black hole as a whole. The latter condition implies that the SMBH mass is bounded from above by the Hill's mass ${ }^{1}: M \lesssim M_{\mathrm{H}} \approx 4 \times 10^{7} M_{\odot}$, for a solar-type star being disrupted (see also ref. ${ }^{31}$ for a more detailed discussion based on TDE demographics). When modelling a TDE emission, an upper bound on the total energy is given by the rest energy of the disrupted star, $E_{\max } \approx M_{\odot} c^{2} \approx 1.8 \times 10^{54}$ erg for a solar-mass star. A useful benchmark parameter is the SMBH Eddington luminosity: $L_{\mathrm{Edd}} \approx 1.3 \times 10^{44} \mathrm{erg} \mathrm{s}^{-1}\left(M /\left(10^{6} M_{\odot}\right)\right)$.

The Blandford-Znajek mechanism ${ }^{32}$ suggests that a weak initial magnetic field in the accretion disk in combination with a high black-hole spin can lead to the formation of a jet. Numerical simulations of TDEs that are based on general relativistic radiation magnetohydrodynamics confirm this hypothesis; see, in particular, the unified model in ref. ${ }^{24}$, where a relatively high spin and $M=5 \times 10^{6} M_{\odot}$ were used. This simulation obtains an average mass accretion rate (at near-peak times) $\dot{M} \approx 10^{2} L_{\text {Edd }}$ (see also refs. ${ }^{33,34}$ ), of which $\sim 20 \%$ and $\sim 3 \%$ go into the jet and the bolometric luminosity, respectively (a remaining $20 \%$ powers the outflow). These fractions result in a moderately super-Eddington jet, and a total radiative emission near the Eddington limit (assuming the results of ref. ${ }^{24} \mathrm{can}$ be rescaled for black holes of different masses):

$$
\begin{gathered}
L_{\text {jet }}^{\text {phys }} \approx 20 L_{\mathrm{Edd}} \approx 3 \times 10^{45} \mathrm{erg} \mathrm{s}^{-1}\left(\frac{M}{10^{6} M_{\odot}}\right) ; \\
L_{\mathrm{bol}} \approx 3 L_{\mathrm{Edd}} \approx 4 \times 10^{44} \mathrm{erg} \mathrm{s}^{-1}\left(\frac{M}{10^{6} M_{\odot}}\right),
\end{gathered}
$$

where $L_{\text {jet }}^{\text {phys }}$ is the physical jet luminosity and $L_{\text {bol }}$ is the bolometric luminosity. In ref. ${ }^{24}$, the density profile of the accretion disk was modelled, indicating that the typical size of the optically thick region (that is, the radial distance where the optical depth for electron scattering is equal to unity) is

$$
R_{\mathrm{BB}} \approx 10^{3} R_{\mathrm{s}} \approx 3 \times 10^{14} \mathrm{~cm}\left(\frac{M}{10^{6} M_{\odot}}\right),
$$

approximately, and for $M \approx 10^{6} M_{\odot}$ (the validity over wide ranges of $M$ has not been studied).

The velocity profile of the gas indicated increasingly fast outflows in regions of decreasing density (away from the plane of the accretion disk and closer to the jet), with speeds reaching $v \approx 0.1 c$ or even $v \approx 0.5 c$.

From a comparison with the measured parameters of AT2019dsg, an overall consistency appears. We note in particular the good agreement of the $\mathrm{BB}$ luminosity and radius (or, in other words, of the measured BB luminosity and temperature, via the Stefan-Boltzmann law, from which we find $R_{\mathrm{BB}}=\left(L_{\mathrm{BB}} / 4 \pi \sigma_{\mathrm{SB}} T_{\mathrm{BB}}^{4}\right)^{1 / 2} \approx 4 \times 10^{14} \mathrm{~cm}$, where $\sigma_{\mathrm{SB}}$ is the Stefan-Boltzmann constant, in agreement with the value quoted in ref. ${ }^{18}$ ) with equations (3) and (4), which indicate a black hole mass $M \approx 10^{6} M_{\odot}$ for AT2019dsg. This value of $M$ also ensures basic consistency with the measured $\mathrm{X}$-ray emission radius, which 
is found to be up to a factor of a few larger than $R_{\mathrm{s}}$ (see, for example, the NICER measurement, $r_{\mathrm{X}}=6.8(+0.9,-0.7) 10^{11} \mathrm{~cm}\left(\right.$ ref. ${ }^{22}$ ), and might be underestimated due to observational effects ${ }^{17}$. We note that higher values of the SMBH mass, $M \approx 10^{7} M_{\odot}$, are obtained using the empirical SMBH-galaxy bulge mass correlation; see, for example, ref. ${ }^{35}$; however, a TDE-specific relationship, which includes TDE demographics, indicates scattering around $M \approx 10^{6} M_{\odot}$ for that bulge mass ${ }^{36}$. A new method based on TDE dynamics ${ }^{37}$ gives an estimate of $M \approx 1.3 \times 10^{6} M_{\odot}$ for AT2019dsg, consistent with our choice. We stress that should a higher black-hole mass be established in the future, our model would remain valid, although with modified parameters. For instance, one may assume that a smaller fraction of the total energy goes into the jet (at the expense of a physical jet luminosity below the Eddington luminosity), or a corresponding increase of the physical jet luminosity potentially coming with enhanced neutrino production (at the expense of increased tension with signatures of the jet).

Moving now to describing the long-term evolution $\left(t-t_{\text {peak }} \gtrsim 10 \mathrm{~d}\right)$ of a TDE signal, we note that no detailed numerical modelling exists, so far. Therefore, this part of the signal is more open to speculation and variety of interpretation. Here we adopt $L_{\mathrm{BB}}$ as a quantity of particular relevance, as it is probably a direct indicator of the accretion disk formed by the debris of the disrupted star. We model the time evolution of $L_{\mathrm{BB}}$ following ref. ${ }^{18}$, with a change from faster to slower cooling at $t-t_{\text {peak }} \gtrsim 100 \mathrm{~d}$ (Fig. 1), where the cooling rate approaches the trend $L_{\mathrm{BB}} \propto t^{-5 / 3}$, which is expected if the mass accretion rate follows the fallback time of stellar material ${ }^{1,2}$ (see dotted curve in Fig. 1).

Our proposed jetted TDE scenario builds on the methods of ref. ${ }^{11}$. In the remainder of this section, the main features, assumptions and inputs are described.

(1) Jet variability, Lorentz factor and physical energy. For the jet, a bulk Lorentz factor $\Gamma \approx \mathcal{O}(10)$ is a natural value, inspired by AGN observations (for example, ref. ${ }^{38}$ ) and consistent with the best-known jetted TDE, Swift J1644 $+57^{39}$. We take $\Gamma=7$, and assume a viewing angle of zero, therefore the Doppler factor is $D=2 \Gamma \approx 14$; these values are centred around the usual assumption of a boost factor of about 10 . Consequently, the jet opening angle can be estimated as $1 / \Gamma$. Matter propagating in the jet has density and velocity inhomogeneities, leading to collisions of plasma shells at the collision radius $R_{\mathrm{C}}$ where internal shocks form, and proton acceleration and subsequent neutrino production via proton-photon scattering occurs. The inhomogeneities are characterized by the variability timescale of the jet, $t_{v}$, for which the Schwarzschild time is a plausible lower limit: $t_{\mathrm{v}} \gtrsim \tau_{\mathrm{s}} \approx 2 \pi R_{\mathrm{s}} / c \approx 63 \mathrm{~s}\left(M /\left(10^{6} M_{\odot}\right)\right)$. A comparable value, $t_{\mathrm{v}} \approx 100 \mathrm{~s}$, was favoured by the Swift J1644+57 data ${ }^{39}$, and is adopted here. Using the estimates above for $\Gamma$ and $t_{\mathrm{v}}$, one obtains a typical $R_{\mathrm{C}} \approx 2 \Gamma^{2} c t_{\mathrm{v}} \gtrsim 2 \Gamma^{2} c \tau_{\mathrm{s}}$ of about a few $\times 10^{14} \mathrm{~cm}$. Note how this value is comparable to $R_{\mathrm{BB}}$, equation (4).

For the physical energy of the jet, we assume $L_{\text {jet }}^{\text {phys }}=3 \times 10^{45} \mathrm{erg} \mathrm{s}^{-1} \approx 20 L_{\mathrm{Edd}}$ at peak time, consistent with equation (2). We also assume that $L_{\text {jet }}^{\text {phys }}$ evolves with time proportionally to $L_{\mathrm{BB}}$ until when $L_{\text {jet }}^{\text {phys }}$ drops below $L_{\mathrm{Edd}}$ and the jet is expected to cease $\mathrm{e}^{2}$. The time of jet cessation depends on the (uncertain) evolution of $R_{\mathrm{BB}}$, and can take place at $\sim 170$ to $300 \mathrm{~d}$ post peak (Fig. 1 ) — which is in any case after the time of the neutrino detection; we apply an exponential cutoff $\propto \exp \left(-L_{\text {edd }} / L_{\text {jet }}^{\text {phys }}\right)$ to the proton luminosity to include this effect. It can be estimated that over this timescale a total emitted energy $E_{\text {tot }} \lesssim 3 \times 10^{-2} M_{\odot} c^{2}$ is needed to power the jet. We assume that electromagnetic signatures of the jet cannot be seen due to absorption, similarly to the case of X-rays (discussed below)

(2) Collision radius. The long delay of the neutrino detection with respect to $t_{\text {peak }}$ suggests little or no decrease of the neutrino luminosity over more than $100 \mathrm{~d}$. To reproduce this feature, we introduce a new element, a time-decreasing collision radius $R_{\mathrm{C}}$. In particular, inspired by the numerical similarity $R_{\mathrm{C}} \approx R_{\mathrm{BB}}$ at peak time, we assume that $R_{C}$ follows the observed evolution of $R_{\mathrm{BB}}$ (ref. ${ }^{18}$ ):

$$
\frac{R_{\mathrm{C}}}{10^{14} \mathrm{~cm}} \approx \begin{cases}5.0 \exp \left(-\frac{t-t_{\text {peak }}}{109 \mathrm{~d}}\right), & t-t_{\text {peak }} \leq 150 \mathrm{~d} \\ 1.3, & t-t_{\text {peak }}>150 \mathrm{~d} .\end{cases}
$$

In general, a time-decreasing $R_{\mathrm{C}}$ can be justified in the context of the overall decline of the power of the jet, which might result for example, in a decreasing value of $\Gamma$. We note that the estimate $R_{\mathrm{C}} \approx 2 \Gamma^{2} t_{\mathrm{v}}$ does not literally hold in multizone collision models, but rather a more physical description of the collision radius should be done in terms of the distance between the plasma shells and their width (see ref. ${ }^{40}$ for an in-depth discussion). Since the pion production efficiency scales $\propto R_{\mathrm{C}}^{-2}$, the drop in $R_{\mathrm{C}}$ will enhance the late-term neutrino production.

(3) Target photons. Another key element of our model is that the background photons necessary for the photo-pion production originate externally to the jet, as the $\mathrm{X}$-rays that are emitted from the inner accretion disk (at $R \approx R_{\mathrm{X}}$ ) are then backscattered into the jet funnel (Fig. 2, right panel). This assumption is attractive because it links the neutrino production to AT2019dsg being particularly bright in X-rays. The description also naturally fits the neutrino energy, as the target photon energy to produce petaelectronvolt neutrinos can be estimated (for external photons boosted into the jet frame) as

$$
E_{\mathrm{X}}(\mathrm{keV}) \approx \frac{0.025}{E_{\nu}(\mathrm{PeV})}
$$

Therefore, for the jetted TDE scenario with external radiation, X-rays with the observed temperature are the ideal target. In principle, some UV photons could also reach the collision region and serve as targets for neutrino production. However, their contribution should be negligible, because: (i) the flux of backscattered UV photons should be small, since observations are consistent with an unabsorbed UV flux and the emission geometry is different (see (4) for their potential impact); and (ii) unscattered UV photons would enter the (relativistically moving) collision region from behind, resulting in decreasing photo-pion efficiency.

The observed exponential decline of $L_{\mathrm{X}}$ suggests that a time-dependent absorption effect might be at play. Hence, we consider a scenario where an expanding outflow obscures the X-rays. For an expansion speed $v \approx 0.1 c$-which is conservative, values reaching $v \approx 0.5 c$ are expected closer to the jet (see ref. ${ }^{24}$ )we find that, over the characteristic X-ray decline time of $\sim 10 \mathrm{~d}$, the cocoon expands out to at least a distance $\sim 3 \times 10^{15} \mathrm{~cm}$, which can serve as an estimate for the absorption radius $R_{\mathrm{abs}}$. Considering that $R_{\mathrm{abs}}$ exceeds the initial value of $R_{\mathrm{C}}$ by nearly an order of magnitude, it is realistic to expect that a fraction of the X-ray photons will be absorbed/reprocessed over the length scale $R_{\mathrm{C}} \approx R_{\mathrm{BB}}$. The scattered photons will then serve as an external target photon field of isotropized X-rays, leading to Doppler-boosted (by a factor $D^{2}$, leading to enhanced pion production) target photon density similar to external photon targets in AGNs (see, for example, ref. ${ }^{41}$, whose description we follow here).

One can check that our proposed mechanism for the photon background is compatible with theoretical outflow models. Considering that in such models the matter density has a somewhat complicated dependence on radial distance and on time, and that several processes contribute to the scattering and absorption of X-rays ${ }^{42}$, only a rough estimate can be presented here. The Thomson optical depth (from charge neutrality, assuming that the electron density is half of that of protons/neutrons and there is a significant contribution from free electrons) is given by

$$
\tau_{\mathrm{T}} \approx 2\left(\frac{\rho}{10^{-14} \mathrm{~g} \mathrm{~cm}^{-3}}\right)\left(\frac{d}{10^{15} \mathrm{~cm}}\right),
$$

where $d$ is the travelled distance. In ref. ${ }^{24}$ (see Fig. 4 there; for the densities $\rho$, see Fig. 3), the numerically calculated region where the electron-scattering optical depth approaches 1 is shown, and its size is found to be comparable to $R_{\mathrm{BB}}$ (for a wide range of angular distances from the jet funnel), which justifies our assumption of photon isotropization at and beyond that scale of length. Note that the photon absorption opacity especially increases beyond the photo-ionization threshold of hydrogen $(13.6 \mathrm{eV})$, which means that it is plausible that UV photons can escape whereas X-rays are confined; details are model dependent.

To implement the scenario described above quantitatively, we model the unattenuated X-ray luminosity according to simulations for TDEs with slim disks, for example, ref. ${ }^{43}$, which show that the X-ray luminosity does not follow the mass fallback rate, but stays nearly constant up to the time of flare cessation (the mass accretion rate becomes sub-Eddington). In ref. ${ }^{43}$, an exponential drop over a timescale of $200 \mathrm{~d}$ post peak is found for the SMBH mass used here, which we incorporate into our model, assuming that the unattenuated light curve is at the level of the observations at the earliest times measurements are available $\left(t-t_{\text {peak }}=17 \mathrm{~d}\right)$. Note that the applicability of the slim-disk model may be limited, especially at early times ${ }^{43}$. However, our neutrino light curve does not qualitatively change even if the $\mathrm{X}$-ray luminosity follows the $\mathrm{BB}$ one. We furthermore assume that $10 \%$ of the unattenuated $\mathrm{X}$-rays isotropize and build up on the attenuation timescale, with the same energy spectrum as the unattenuated parent photon flux (which is plausible considering the relatively low rate or photon re-processing). Note that this radiation will not be observable, so any late-term X-ray bounds only apply to the thick blue (dashed) curve in Fig. 1.

(4) Hadronic content of the jet. Protons (proton energy: $E_{\mathrm{p}}^{\prime}$ ) are assumed to be accelerated at the collision radius $R_{\mathrm{C}}$ by internal shocks to a power-law spectrum $\propto E_{\mathrm{p}}^{\prime-2}$ (primed indices refer to the shock frame) with a maximal energy determined by balancing the acceleration rate $t_{\mathrm{acc}}^{\prime-1}=\eta c / R_{\mathrm{L}}^{\prime}$ (with moderate acceleration efficiency $\eta=0.01$ and $R_{\mathrm{L}}^{\prime}$ the Larmor radius of the proton) with the synchrotron loss and dynamical rates (so the Hillas criterion is satisfied). As the interactions occur in the optically thin (to $p \gamma$ interactions) regime, the requirements for proton acceleration are moderate. The (isotropic equivalent) proton luminosity is given by $L_{\mathrm{p}}^{\text {iso }} \approx\left(2 \Gamma^{2}\right) \varepsilon L_{\text {jet }}^{\text {phys }}$ (Fig. 1$)$, where $\left(2 \Gamma^{2}\right)$ is the beaming factor and $\varepsilon$ is the transfer (dissipation) efficiency from jet kinetic energy into non-thermal radiation dominated by baryons. We take $\varepsilon \approx 0.2$, which is well within the range of typical values for gamma-ray burst internal shock scenarios (see, for example, refs. ${ }^{40,44-47}$ ).

Note that, if the non-thermal radiation from the jet can escape, its non-observation results in a lower limit on the jet baryonic loading, $\xi_{\mathrm{b}}$, which is usually defined as the ratio of proton and electron injection luminosities. More specifically, assuming that the electrons are in equilibrium with the X-rays, one can estimate the minimal baryonic loading by comparing the required isotropic-equivalent proton luminosity to the X-ray bounds. Using the late-term bound $L_{\mathrm{X}} \lesssim 10^{42} \mathrm{erg} \mathrm{s}^{-1}$ (ref. ${ }^{18}$ ) one obtains $\xi_{\mathrm{b}} \gtrsim 10^{3}-10^{4}$, which implies that the expected non-thermal X-ray signal is below the current bound if there is sufficient 
energy in protons compared with electrons. Comparable or larger numbers are typical for hadronic models of AGNs, such as the first identified neutrino emitter, TXS $0506+056^{48}$. One should consider, however, that constraints depend on the photon spectrum of AT2019dsg. For example, if an AGN-like spectrum is assumed-with its characteristic suppression in the X-ray band and likely peak in the optical-UV and gamma-ray ranges - the constraint on $\xi_{\mathrm{b}}$ may be about an order of magnitude weaker. One may wonder about the contribution of non-thermal X-rays from the jet to the neutrino production. Because these photons are produced in the jet co-moving frame (and so are not Lorentz boosted), for them the neutrino production efficiency is lower compared with the external (boosted) photons; this efficiency problem has been studied for TXS 0506+056 and AGNs in general ${ }^{49,50}$.

(5) Magnetic field and other assumptions. We assume that the magnetic field energy density takes a fraction of $10 \%$ of the proton energy density ${ }^{11}$ (corresponding to $2 \%$ of the jet kinetic energy), which leads to a magnetic field $B^{\prime} \approx 90 \mathrm{G}$. The neutrino mixing angles are taken from from NuFIT $4.1(2019)^{51}$ Note that in our model, the information from radio data are not directly relevant for the neutrino production, mainly because $R_{\text {radio }} \gg R_{C}$.

Our numerical treatment closely follows ref. ${ }^{11}$, with the time evolution of the spectra being calculated in discrete steps of $1 \mathrm{~d}$ width.

(6) Shape of the resulting neutrino spectrum and light curve. The neutrino light curve (Fig. 1) is approximately described by $L_{\nu} \propto L_{\mathrm{p}}^{\text {iso }} f_{\nu}$, where $f_{\nu} \propto L_{\mathrm{X}}^{\text {isotropized }} / R_{\mathrm{C}}^{2}$ is the neutrino production efficiency and $L_{\mathrm{X}}^{\text {isotropized }}$ is the luminosity of the isotropized $\mathrm{X}$-rays. Consequently, there are three competing processes determining its shape: (1) the evolution of $L_{\mathrm{p}}^{\text {iso }}$, dropping with the $\mathrm{BB}$ luminosity (Fig. 1), (2) the decrease of $R_{\mathrm{C}}$ following equation (5) and (3) the evolution of $L_{\mathrm{X}}^{\text {isotropized }}$. The time of the first peak of the neutrino light curve is determined by the timescale of isotropization of the $\mathrm{X}$-rays, which is inferred from the observed obscuration timescale. The decline following the first peak comes from the combined effect of decreasing proton luminosity-including the break at $t-t_{\text {peak }} \approx 100 \mathrm{~d}-$ and of the decreasing collision radius. The peak at $t-t_{\text {peak }} \approx 150 \mathrm{~d}$ originates from the stagnation of the collision radius decrease in equation (5). The later decline, after the second peak, follows the decreasing $L_{\mathrm{p}}^{\text {iso }}$ and its eventual exponential cutoff, occurring when $L_{\text {jet }}^{\text {phys }}$ falls below the Eddington luminosity (at $t-t_{\text {peak }} \approx 300 \mathrm{~d}$, shown in Fig. 1). Note that following the slim-disk model, the unattenuated X-ray luminosity is assumed to decline exponentially over a timescale of $200 \mathrm{~d}$. The effect of that model-dependent time evolution on the neutrino light curve is secondary: as a test, we produced results for an alternative scenario, where the unattenuated X-ray luminosity evolves with time following the $\mathrm{BB}$ luminosity; we found that the qualitative conclusions are robust.

Let us now comment on the neutrino spectrum in Fig. 3. Its general form roughly matches the naive estimate presented in equation (6) for the $\Delta$-resonance; however, its detailed shape is broader, because multipion production is efficient due to the high target photon temperature. A number of uncertainties affect the neutrino spectrum and light curve. Specifically, the high-energy end of the spectrum depends on the maximal proton energy (determined by the acceleration efficiency), $E_{\mathrm{p}, \max } \gtrsim 20 E_{\nu} \approx 4 \mathrm{PeV}$ (estimated from the observed neutrino energy $E_{\nu} \approx 0.2 \mathrm{PeV}$ ). The neutrino spectrum would be enhanced at high energy for higher $E_{\mathrm{p}, \max }$, if a background of lower-energy photon targets were available, for example, as isotropized UV photons. In such a case, the neutrino spectrum would peak at about a factor of 20 higher energy according to equation (6), which implies that $E_{\mathrm{p}, \max } \gtrsim 80 \mathrm{PeV}$ is required. Conservatively, we did not include this possibility in the main text as there is no observational evidence for UV obscuration/backscattering. Moreover, due to the different sizes of the X-ray and UV photospheres, one can not apply the same assumptions as for the X-ray backscattering (thus introducing further uncertainty). Still, we have done that in a test run-assuming the same acceleration efficiency as in the main text-with the goal of studying an extreme scenario. As expected, we find an overall more energetic neutrino spectrum, which would be less compatible with the observed neutrino energy. Note that unscattered UV photons or X-rays (which may be relevant at early times, before obscuration sets in) have an $\sim 10^{2}$ lower Doppler factor than backscattered photons, since they come from behind the production region. Therefore, their contribution to the neutrino flux is suppressed.

\section{Data availability}

The data that support the plots within this paper and other findings of this study are available in the supplementary information or from the corresponding author upon reasonable request. Source data are provided with this paper.

\section{Code availability}

The codes that support the plots within this paper and other findings of this study are available from the corresponding author upon reasonable request.

Received: 26 May 2020; Accepted: 8 January 2021; Published online: 22 February 2021

\section{References}

1. Hills, J. G. Possible power source of Seyfert galaxies and QSOs. Nature 254, 295-298 (1975).

2. Rees, M. J. Tidal disruption of stars by black holes of 10 to the 6 th-10 to the 8th solar masses in nearby galaxies. Nature 333, 523-528 (1988).

3. Lacy, J. H., Townes, C. H. \& Hollenbach, D. J. The nature of the central parsec of the Galaxy. Astrophys. J. 262, 120-134 (1982).

4. Phinney, E. S. in The Center of the Galaxy (ed. Morris, M.) 543-553 (Springer, 1989).

5. Farrar, G. R. \& Gruzinov, A. Giant AGN flares and cosmic ray bursts. Astrophys. J. 693, 329-332 (2009).

6. Farrar, G. R. \& Piran, T. Tidal disruption jets as the source of ultra-high energy cosmic rays. Preprint at https://arxiv.org/abs/1411.0704 (2014).

7. Wang, X.-Y., Liu, R.-Y., Dai, Z.-G. \& Cheng, K. Probing the tidal disruption flares of massive black holes with high-energy neutrinos. Phys. Rev. D 84, 081301 (2011).

8. Wang, X.-Y. \& Liu, R.-Y. Tidal disruption jets of supermassive black holes as hidden sources of cosmic rays: explaining the IceCube $\mathrm{TeV}-\mathrm{PeV}$ neutrinos. Phys. Rev. D 93, 083005 (2016).

9. Dai, L. \& Fang, K. Can tidal disruption events produce the IceCube neutrinos? Mon. Not. R. Astron. Soc. 469, 1354-1359 (2017).

10. Senno, N., Murase, K. \& Meszaros, P. High-energy neutrino flares from X-ray bright and dark tidal disruption events. Astrophys. J. 838, 3 (2017).

11. Lunardini, C. \& Winter, W. High energy neutrinos from the tidal disruption of stars. Phys. Rev. D 95, 123001 (2017).

12. Guépin, C., Kotera, K., Barausse, E., Fang, K. \& Murase, K. Ultra-high energy cosmic rays and neutrinos from tidal disruptions by massive black holes. Astron. Astrophys. 616, A179 (2018)

13. Biehl, D., Boncioli, D., Lunardini, C. \& Winter, W. Tidally disrupted stars as a possible origin of both cosmic rays and neutrinos at the highest energies. Sci. Rep. 8, 10828 (2018).

14. Hayasaki, K. \& Yamazaki, R. Neutrino emissions from tidal disruption remnants. Astrophys. J. 886, 114 (2019).

15. The IceCube Collaboration. Search for neutrinos from populations of optical transients. In Proc. 36th International Cosmic Ray Conference (ICRC2019) 1016 (PoS, 2019).

16. Aartsen, M. et al. IceCube-191001A-IceCube observation of a high-energy neutrino candidate event. GRB Coord. Netw. 25913 (2019).

17. Stein, R. et al. A tidal disruption event coincident with a high-energy neutrino. Nat. Astron. https://doi.org/10.1038/s41550-020-01295-8 (2021).

18. van Velzen, S. et al. Seventeen tidal disruption events from the first half of ZTF survey observations: entering a new era of population studies. Preprint at https://arxiv.org/abs/2001.01409 (2020).

19. Perez-Torres, M. et al. Unambiguous radio detection of the tidal disruption event AT2019dsg with e-MERLIN. The Astronomer's Telegram 12960 (2019).

20. Sfaradi, I. et al. A possible radio detection of the TDE candidate AT2019DSG by AMI-LA. The Astronomer's Telegram 12798 (2019).

21. Pasham, D., Remillard, R. \& Wevers, T. Swift discovers X-rays from the newly discovered tidal disruption flare candidate AT2019dsg. The Astronomer's Telegram 12777 (2019)

22. Pasham, D. et al. NICER X-ray observations of the young tidal disruption flare candidate AT2019dsg. The Astronomer's Telegram 12825 (2019).

23. Lee, C.-H. et al. Optical polarimetry of the tidal disruption event AT2019dsg. Astrophys J. 892, L1 (2020).

24. Dai, L., McKinney, J. C., Roth, N., Ramirez-Ruiz, E. \& Miller, M. C. A unified model for tidal disruption events. Astrophys. J. Lett. 859, L20 (2018).

25. Hummer, S., Ruger, M., Spanier, F. \& Winter, W. Simplified models for photohadronic interactions in cosmic accelerators. Astrophys. J. 721, 630-652 (2010).

26. Strotjohann, N. L., Kowalski, M. \& Franckowiak, A. Eddington bias for cosmic neutrino sources. Astron. Astrophys. 622, L9 (2019).

27. Generozov, A. et al. The influence of circumnuclear environment on the radio emission from TDE jets. Mon. Not. R. Astron. Soc. 464, 2481-2498 (2017).

28. Alexander, K. D., van Velzen, S., Horesh, A. \& Zauderer, B. A. Radio properties of tidal disruption events. Space Sci. Rev. 216, 81 (2020).

29. Fang, K., Metzger, B. D., Vurm, I., Aydi, E. \& Chomiuk, L. High-energy neutrinos and gamma rays from nonrelativistic shock-powered transients Astrophys. J. 904, 4 (2020).

30. Murase, K., Kimura, S. S., Zhang, B. T., Oikonomou, F. \& Petropoulou, M. High-energy neutrino and gamma-ray emission from tidal disruption events. Astrophys. J. 902, 108 (2020).

31. Kochanek, C. Tidal disruption event demographics. Mon. Not. R. Astron. Soc. 461, 371-384 (2016).

32. Blandford, R. \& Znajek, R. Electromagnetic extractions of energy from Kerr black holes. Mon. Not. R. Astron. Soc. 179, 433-456 (1977).

33. De Colle, F., Guillochon, J., Naiman, J. \& Ramirez-Ruiz, E. The dynamics, appearance and demographics of relativistic jets triggered by tidal disruption of stars in quiescent supermassive black holes. Astrophys. J. 760, 103 (2012). 
34. Guillochon, J. \& Ramirez-Ruiz, E. Hydrodynamical simulations to determine the feeding rate of black holes by the tidal disruption of stars: the importance of the impact parameter and stellar structure. Astrophys. J. 767, 25 (2013).

35. McConnell, N. J. \& Ma, C.-P. Revisiting the scaling relations of black hole masses and host galaxy properties. Astrophys. J. 764, 184 (2013).

36. Wevers, T. et al. Black hole masses of tidal disruption event host galaxies II. Mon. Not. R. Astron. Soc. 487, 4136-4152 (2019).

37. Ryu, T., Krolik, J. \& Piran, T. Measuring stellar and black hole masses of tidal disruption events. Astrophys. J. 904, 73 (2020).

38. Chai, B., Cao, X. \& Gu, M. What governs the bulk velocity of the jet components in active galactic nuclei? Astrophys. J. 759, 114 (2012).

39. Burrows, D. et al. Relativistic jet activity from the tidal disruption of a star by a massive black hole. Nature 476, 421-424 (2011).

40. Bustamante, M., Murase, K., Winter, W. \& Heinze, J. Multi-messenger light curves from gamma-ray bursts in the internal shock model. Astrophys. J. 837, 33 (2017).

41. Murase, K., Inoue, Y. \& Dermer, C. D. Diffuse neutrino intensity from the inner jets of active galactic nuclei: impacts of external photon fields and the blazar sequence. Phys. Rev. D 90, 023007 (2014).

42. Roth, N., Kasen, D., Guillochon, J. \& Ramirez-Ruiz, E. The X-ray through optical fluxes and line strengths of tidal disruption events. Astrophys. J. 827, 3 (2016).

43. Wen, S., Jonker, P. G., Stone, N. C., Zabludoff, A. I. \& Psaltis, D. Continuum-fitting the X-ray spectra of tidal disruption events. Astrophys. J. 897, 80 (2020)

44. Pe'er, A. Physics of gamma-ray bursts prompt emission. Adv. Astron. 2015 , 907321 (2015).

45. Sari, R. \& Piran, T. Variability in GRBs: a clue. Astrophys. J. 485, 270 (1997).

46. Kino, M., Mizuta, A. \& Yamada, S. Hydrodynamical effects in internal shock of relativistic outflows. Astrophys. J. 611, 1021-1032 (2004).

47. Rudolph, A., Heinze, J., Fedynitch, A. \& Winter, W. Impact of the collision model on the multi-messenger emission from gamma-ray burst internal shocks. Astrophys. J. 893, 72 (2020)

48. Gao, S., Fedynitch, A., Winter, W. \& Pohl, M. Modelling the coincident observation of a high-energy neutrino and a bright blazar flare. Nat. Astron. 3, 88-92 (2019).

49. Keivani, A. et al. A multimessenger picture of the flaring blazar TXS 0506+056: implications for high-energy neutrino emission and cosmic ray acceleration. Astrophys. J. 864, 84 (2018).
50. Rodrigues, X., Gao, S., Fedynitch, A., Palladino, A. \& Winter, W. Leptohadronic blazar models applied to the 2014-2015 flare of TXS 0506+056. Astrophys. J. Lett. 874, L29 (2019).

51. Esteban, I., Gonzalez-Garcia, M., Hernandez-Cabezudo, A., Maltoni, M. \& Schwetz, T. Global analysis of three-flavour neutrino oscillations: synergies and tensions in the determination of $\theta_{23}, \delta_{C P}$, and the mass ordering. J. High Energy Phys. 2019, 106 (2019).

52. Blaufuss, E., Kintscher, T., Lu, L. \& Tung, C. F. The next generation of IceCube real-time neutrino alerts. In Proc. 36th International Cosmic Ray Conference (ICRC2019) 1021 (PoS, 2020).

53. Aartsen, M. et al. Searches for extended and point-like neutrino sources with four years of IceCube data. Astrophys. J. 796, 109 (2014).

\section{Acknowledgements}

We thank A. Franckowiak, M. Kowalski, R. Stein, A. Taylor and S. van Velzen for useful discussions. This work has been supported by the European Research Council (ERC) under the European Unions Horizon 2020 research and innovation programme (grant number 646623), and by the US National Science Foundation grant number PHY-1613708.

\section{Author contributions}

The theoretical ideas were equally developed by C.L. and W.W. Numerical simulations were performed by W.W. The artwork was produced by C.L. and the results figures by W.W. Both authors contributed equally to the manuscript writing.

\section{Competing interests}

The authors declare no competing interests.

\section{Additional information}

Supplementary information The online version contains supplementary material available at https://doi.org/10.1038/s41550-021-01305-3.

Correspondence and requests for materials should be addressed to W.W.

Peer review information Nature Astronomy thanks Francis Halzen, Kikitake Hayasaki and the other, anonymous, reviewer(s) for their contribution to the peer review of this work.

Reprints and permissions information is available at www.nature.com/reprints.

Publisher's note Springer Nature remains neutral with regard to jurisdictional claims in published maps and institutional affiliations.

(c) The Author(s), under exclusive licence to Springer Nature Limited 2021, corrected publication 2021 